\title{
STABLE DETERMINATION OF A CRACK IN A PLANAR INHOMOGENEOUS CONDUCTOR*
}

\author{
GIOVANNI ALESSANDRINI ${ }^{\dagger}$ AND LUCA RONDI ${ }^{\ddagger}$
}

\begin{abstract}
We prove a stability estimate for the inverse problem of cracks under essentially minimal regularity assumptions on the crack and on the background conductivity.
\end{abstract}

Key words. inverse problems, cracks, elliptic equations, quasi-conformal mappings

AMS subject classifications. 35R30, 78A30, 31A25, 30C62

PII. S0036141097325502

1. Introduction. We consider the problem of determining a crack in an electrically conducting body from current and voltage measurements at the boundary. The mathematical theory for this inverse problem was initiated by A. Friedman and $\mathrm{M}$. Vogelius $[\mathrm{F}-\mathrm{V}]$, who proved uniqueness theorems for a crack in a planar conductor. Stability estimates were obtained in [A2], [A3], [DV] for the case of a single crack in a homogeneous isotropic planar conductor. For an extended account on the results for this problem and for further references the reader is referred to [A-DB], where a three-dimensional theory for this problem is developed.

In this paper we prove a stability estimate for the determination of a crack in an inhomogeneous planar conductor under essentially minimal regularity assumptions on the (unknown) crack and on the (known) background conductivity.

We shall consider the conductor $\Omega$ as a simply connected bounded domain in the plane with Lipschitz boundary. The conductivity within $\Omega$ is given by a bounded and measurable tensor $A$ which satisfies a uniform ellipticity condition. A crack $\sigma$ in $\Omega$ will be a simple open curve within $\Omega$ which we shall a priori assume to be Lipschitz. Given a zero average function $\psi$ on $\partial \Omega$, representing the prescribed current density, the electrostatic potential $u$ in $\Omega$ will be, in the presence of the crack $\sigma$, the weak solution of the following (direct) Neumann boundary value problem:

$$
\begin{cases}\operatorname{div}(A \nabla u)=0 & \text { in } \Omega \backslash \sigma, \\ A \nabla u \cdot \nu=0 & \text { on either side of } \sigma, \\ A \nabla u \cdot \nu=\psi & \text { on } \partial \Omega,\end{cases}
$$

where $\nu$ denotes the unit normal with outward orientation when on $\partial \Omega$.

The inverse problem consists of determining the crack $\sigma$ from the voltage measurements $\left.u\right|_{\Sigma}, \Sigma$ being a portion of $\partial \Omega$, corresponding to one or more prescribed current densities $\psi$.

Notice that this model corresponds to the so-called case of a perfectly insulating crack; let us stress here that our present method would enable us also to treat, with analogous results, the so-called case of perfectly conducting cracks. For the sake of brevity, we shall not discuss this case any further.

\footnotetext{
* Received by the editors August 4, 1997; accepted for publication January 27, 1998; published electronically December 11, 1998.

http://www.siam.org/journals/sima/30-2/32550.html

${ }^{\dagger}$ Dipartimento di Scienze Matematiche, Università degli Studi di Trieste, piazzale Europa 1, 34100 Trieste, Italy (alessang@univ.trieste.it). The work of this author was partially supported by MURST and CNR.

${ }^{\ddagger}$ SISSA-ISAS, via Beirut 2-4, 34014 Trieste, Italy (rondi@sissa.it).
} 
As is well known since the work of A. Friedman and M. Vogelius, in order to uniquely determine $\sigma$ it is necessary to perform measurements for at least two different choices $\psi_{1}, \psi_{2}$ of the current density $\psi$. We shall use here current densities $\psi_{1}, \psi_{2}$ analogous to those used in previous works on uniqueness [Br-V], [A-DV], [K-Se], which can be viewed as general models of a two-electrode configuration in which one electrode is kept fixed and the other is placed in two different locations. See the following section 2 for details.

Our main result (Theorem 2.1) is that the crack $\sigma$ depends continuously on the boundary measurements at a rate which is of log-log type.

That is, we obtain a result which is comparable with those previously obtained for the case of a homogeneous conductor. See the concluding remarks for further details.

The present approach, however, is different from the one in [A2], [A3], [DV], which took advantage of the uniform conductivity by the use of special conformal transformations. Rather, it is closer to the approach used in [A-DV] to prove uniqueness of multiple cracks in an inhomogeneous conductor, the main novelty here being the need of stability estimates for a Cauchy problem for the elliptic equation in (1.1). In fact we shall show that the Cauchy problem for such elliptic equations has a stability character analogous to the one for the Laplace equation regardless of the smoothness of the coefficients. We shall prove this by a generalization of the classical method of harmonic measure, Theorem 4.5. We believe that this result can have some independent interest.

In section 2 we start by listing all the needed a priori assumptions and we state our main Theorem 2.1.

In section 3 we collect results based on the connections between elliptic equations in two variables, first-order Beltrami-type equations, and quasi-conformal mappings. The principal result of this section is contained in Proposition 3.7 stating Hölder continuity properties of the mappings $f, f^{-1}$, where $f$ is given by $f=u+i v, u$ is a solution to (1.1), and $v$ is the associated stream function (i.e., a generalized harmonic conjugate).

Section 4 contains a treatment of a Cauchy problem and its stability properties, the main result for the rest of the paper being Proposition 4.1. Theorem 4.5 is instead a result of general type possibly useful in other contexts.

Section 5 consists of the completion of the proof of Theorem 2.1 and some concluding remarks.

\section{The main theorem.}

Prior information. For every $z=x+i y \in \mathbb{C}$ and for every $r>0$ we denote with $B_{r}(z)$ the disk with center $z$ and radius $r$. As usual, we shall identify complex numbers $z=x+i y \in \mathbb{C}$ with points $(x, y) \in \mathbb{R}^{2}$.

If $\gamma$ is a simple curve (which could be closed) and $z_{0}, z_{1}$ are two points of $\gamma$, we define length ${ }_{\gamma}\left(z_{0}, z_{1}\right)$ the length of the smallest arc in $\gamma$ connecting $z_{0}$ to $z_{1}$.

If $\gamma$ is a simple curve, $r$ is a positive number, and $z$ belongs to $\gamma$, we say that $\gamma \cap B_{r}(z)$ is a Lipschitz graph with norm $M$ if there exists a system of Cartesian coordinates $(x, y)$ with origin in $z$, with respect to which one has

$$
\gamma \cap B_{r}(z)=\left\{(x, y) \mid y=\phi(x), x^{2}+y^{2}<r^{2}\right\}
$$

where $\phi$ is a Lipschitz function on $[-r, r]$ and $\left\|\phi^{\prime}\right\|_{L^{\infty}(-r, r)} \leq M$.

If $\gamma$ is a simple open curve, $r$ is a positive number and $z$ is an endpoint of $\gamma$, we say that $\gamma \cap B_{r}(z)$ is a half Lipschitz graph with norm $M$ if there exists a 
system of Cartesian coordinates $(x, y)$ with origin in $z$ such that with respect to these coordinates one has

$$
\gamma \cap B_{r}(z)=\left\{(x, y) \mid y=\phi(x), 0 \leq x \leq r, x^{2}+y^{2}<r^{2}\right\},
$$

where $\phi$ is a Lipschitz function on $[0, r]$ and $\left\|\phi^{\prime}\right\|_{L^{\infty}(0, r)} \leq M$.

Let $\Omega$ be a bounded domain and $d>0$; we denote

$$
\Omega_{d}=\{z \in \Omega: \operatorname{dist}(z, \partial \Omega)>d\} .
$$

Prior information on the domain. Let $\Omega$ be a bounded, simply connected domain in $\mathbb{R}^{2}$ and let its boundary $\partial \Omega$ be a simple, closed curve satisfying, for given positive constants $L, \delta$, and $M$,

(2.2)(a) perimeter of $\Omega \leq L$,

(2.2)(b) for every $z \in \partial \Omega$; then $\partial \Omega \cap B_{\delta}(z)$ is a Lipschitz graph with norm $M$.

Prior information on the crack. A crack $\sigma$ in $\Omega$ will be a simple, open curve in $\Omega$ such that

(2.3)(a) the length of $\sigma$ is less than $L$;

(2.3)(b) the distance of $\sigma$ from $\partial \Omega$ is $\geq \delta$;

(2.3)(c) if $V_{1}, V_{2}$ are the endpoints of $\sigma$, then for every $i=1,2 \sigma \cap B_{\delta}\left(V_{i}\right)$ is a half Lipschitz graph with norm $M$; furthermore, for any $z \in \sigma \backslash\left(B_{\delta / 2}\left(V_{1}\right) \cup B_{\delta / 2}\left(V_{2}\right)\right)$, $\sigma \cap B_{\delta / 2}(z)$ is a Lipschitz graph with norm $M$.

Prior information on the boundary data. Let $\gamma_{0}, \gamma_{1}, \gamma_{2}$ be three fixed simple arcs in $\partial \Omega$, pairwise internally disjoint.

Given $\Gamma>0$, let us fix three functions $\eta_{0}, \eta_{1}, \eta_{2} \in L^{2}(\partial \Omega)$ such that for every $j=0,1,2$,

(2.4)(a) $\eta_{j} \geq 0$ on $\partial \Omega ; \operatorname{supp}\left(\eta_{j}\right) \subset \gamma_{j}$;

(2.4)(b) $\quad \int_{\partial \Omega} \eta_{j}=1$;

(2.4)(c) $\quad\left\|\eta_{j}\right\|_{L^{2}(\partial \Omega)} \leq \Gamma$.

Then we prescribe the current densities on the boundary $\psi_{1}, \psi_{2}$ to be given by

$$
\psi_{1}=\eta_{0}-\eta_{1}, \quad \psi_{2}=\eta_{0}-\eta_{2} .
$$

We have

(2.6)(a) $\int_{\partial \Omega} \psi_{j}=0$ for every $j=1,2$;

(2.6)(b) $\quad\left\|\psi_{j}\right\|_{L^{2}(\partial \Omega)} \leq 2 \Gamma$ for every $j=1,2$.

Moreover let us consider the following antiderivatives along $\partial \Omega$ of $\psi_{1}, \psi_{2}$ :

$$
\Psi_{j}(s)=\int \psi_{j}(s) \mathrm{d} s, \quad j=1,2,
$$

where the indefinite integral is taken with respect to arclength on $\partial \Omega$ in the counterclockwise direction. The functions $\Psi_{1}, \Psi_{2}$ are defined up to an additive constant.

We remark that from the prior information on $\Omega,(2.2)$, we can find a constant $M_{1}$ depending on $L, \delta$, and $M$ only such that for all $z_{0}, z_{1}$ belonging to $\partial \Omega$ the following inequality holds:

$$
\operatorname{length}_{\partial \Omega}\left(z_{0}, z_{1}\right) \leq M_{1}\left|z_{0}-z_{1}\right| .
$$

Hence $\Psi_{j}$ verify the following property

$$
\left|\Psi_{j}\left(z_{0}\right)-\Psi_{j}\left(z_{1}\right)\right| \leq 2 \Gamma\left(\text { length }_{\partial \Omega}\left(z_{0}, z_{1}\right)\right)^{1 / 2} \leq \Gamma_{1}\left|z_{0}-z_{1}\right|^{1 / 2},
$$

for any $z_{0}, z_{1}$ belonging to the boundary of $\Omega$, where $\Gamma_{1}=2 \Gamma M_{1}^{1 / 2}$. 
Prior information on the conductivity. Given $\lambda, \Lambda>0$, let $A=A(z), z \in \Omega$, be a $2 \times 2$ matrix with bounded measurable entries such that

(2.10)(a) $\quad A(z) \xi \cdot \xi \geq \lambda|\xi|^{2} \quad$ for every $\xi \in \mathbb{R}^{2}$ and for a.e. $z \in \Omega$;

(2.10)(b) $\quad\|A\|_{L^{\infty}(\Omega)} \leq \Lambda$.

For any $i=1,2$, let $u_{i} \in W^{1,2}(\Omega \backslash \sigma)$ be the weak solution of the following Neumann-type boundary value problem:

$$
\begin{cases}\operatorname{div}\left(A \nabla u_{i}\right)=0 & \text { in } \Omega \backslash \sigma, \\ A \nabla u_{i} \cdot \nu=0 & \text { on either side of } \sigma, \\ A \nabla u_{i} \cdot \nu=\psi_{i} & \text { on } \partial \Omega,\end{cases}
$$

where $\nu$ denotes the unit normal, with the outward orientation when on $\partial \Omega$.

That is, we understand that $u_{i}$ satisfies

$$
\int_{\Omega \backslash \sigma} A \nabla u_{i} \cdot \nabla \varphi=\int_{\partial \Omega} \psi_{i} \varphi \quad \text { for every } \varphi \in W^{1,2}(\Omega \backslash \sigma) .
$$

If $\sigma^{\prime}$ is another crack, that is, another curve satisfying conditions (2.3), we denote by $u_{i}^{\prime}$ the solutions to (2.11) when $\sigma$ is replaced with $\sigma^{\prime}$.

We denote by $\Sigma$ a simple arc in $\partial \Omega$ whose length is at least $\delta$.

The set of constants $L, M, \delta, \Gamma, \lambda$, and $\Lambda$ will be referred to as the a priori data. We are now in position to state the main theorem.

THEOREM 2.1. Under the previously stated assumptions, let $\varepsilon>0$ be such that

$$
\max _{i=1,2}\left\|u_{i}-u_{i}^{\prime}\right\|_{L^{\infty}(\Sigma)} \leq \varepsilon
$$

then the two cracks $\sigma, \sigma^{\prime}$ satisfy

$$
\mathrm{d}_{H}\left(\sigma, \sigma^{\prime}\right) \leq \omega(\varepsilon),
$$

where $\omega(\varepsilon)$ is a positive function on $(0,+\infty)$ that verifies

$$
\omega(\varepsilon) \leq K(\log |\log \varepsilon|)^{-\alpha} \text { for every } \varepsilon, 0<\varepsilon<1 / e .
$$

Here $K$ and $\alpha$ are positive constants depending on the a priori data only.

Here $\mathrm{d}_{H}$ denotes the Hausdorff distance. We recall that the Hausdorff distance between bounded closed sets $\sigma$ and $\sigma^{\prime}$ is given by

$$
\mathrm{d}_{H}\left(\sigma, \sigma^{\prime}\right)=\max \left\{\sup _{x \in \sigma^{\prime}} \operatorname{dist}(x, \sigma), \sup _{x \in \sigma} \operatorname{dist}\left(x, \sigma^{\prime}\right)\right\} .
$$

3. Stream functions and quasi-conformal mappings. We begin by reviewing some properties of quasi-conformal mappings which will be used in the sequel.

We shall make repeated use of the following notation for complex derivatives:

$$
f_{\bar{z}}=\frac{1}{2}\left(f_{x}+i f_{y}\right), \quad f_{z}=\frac{1}{2}\left(f_{x}-i f_{y}\right) .
$$
pose.

We denote by $J=\left[\begin{array}{cc}0 & -1 \\ 1 & 0\end{array}\right]$ the counterclockwise rotation of $90^{\circ}$ and by $(\cdot)^{T}$ trans-

Proposition 3.1. Let $D$ be a bounded simply connected domain in $\mathbb{R}^{2}$. Let $A$ satisfy (2.10). Let $u \in W^{1,2}(D)$ be a weak solution to the equation

$$
\operatorname{div}(A \nabla u)=0 \quad \text { in } D .
$$


There exists a function $v \in W^{1,2}(D)$ which satisfies

$$
\nabla v=J A \nabla u \quad \text { almost everywhere in } D .
$$

Moreover, letting $f=u+i v$, we have

$$
f_{\bar{z}}=\mu f_{z}+\nu \overline{f_{z}} \quad \text { almost everywhere in } D,
$$

where $\mu$ and $\nu$ are bounded measurable, complex valued coefficients, satisfying

$$
|\mu|+|\nu| \leq k<1 \quad \text { almost everywhere in } D,
$$

where $k$ is a constant depending on $\lambda, \Lambda$ only.

On the other hand, if $f=u+i v, f \in W^{1,2}(D, \mathbb{C})$, verifies (3.3) with coefficients $\mu$ and $\nu$ satisfying (3.4), then there exists a $2 \times 2$ matrix $A$ such that $u$ is a weak solution of $\operatorname{div}(A \nabla u)=0$ in $D$ and $A$ verifies (2.10) with constants $\lambda, \Lambda>0$ depending upon $k$ only.

The function $v$ appearing above is usually called the stream function associated with $u$. Notice that $v$ is uniquely determined up to an additive constant and also that $v$ is a weak solution to

$$
\operatorname{div}(B \nabla v)=0 \quad \text { in } D
$$

where $B=(\operatorname{det} A)^{-1} A^{T}$.

Proof. For the existence of the stream function $v$ see [A-M, Theorem 2.1]. Then by (3.2), (3.3) follows with $\mu, \nu$ given by

$$
\begin{aligned}
& \mu=\frac{a_{22}-a_{11}-i\left(a_{12}+a_{21}\right)}{a_{11} a_{22}-a_{12} a_{21}+a_{11}+a_{22}+1}, \\
& \nu=\frac{a_{12} a_{21}-a_{11} a_{22}+1+i\left(a_{12}-a_{21}\right)}{a_{11} a_{22}-a_{12} a_{21}+a_{11}+a_{22}+1} .
\end{aligned}
$$

From these expressions and (2.10), one obtains, through elementary although lengthy computations, (3.4).

Conversely, given the coefficients $\mu, \nu$ in (3.3) satisfying (3.4) one obtains (3.1) and (3.2) with $A$ given by

$$
A=\left[\begin{array}{ll}
\frac{|1-\mu|^{2}-|\nu|^{2}}{|1+\nu|^{2}-|\mu|^{2}} & \frac{2 \Im(\nu-\mu)}{|1+\nu|^{2}-|\mu|^{2}} \\
\frac{-2 \Im(\mu+\nu)}{|1+\nu|^{2}-|\mu|^{2}} & \frac{|1+\mu|^{2}-|\nu|^{2}}{|1+\nu|^{2}-|\mu|^{2}}
\end{array}\right]
$$

and the thesis follows.

We recall that a quasi-conformal map $f$ in an open set $D$ is an univalent $W^{1,2}(D, \mathbb{C})$ solution of an equation of the type (3.3), (3.4).

Now we state the following representation theorem, due to L. Bers and L. Nirenberg $[\mathrm{B}-\mathrm{N}]$.

TheOREM 3.2. Let $D \subset B_{1}(0)$, and let $f \in W^{1,2}(D, \mathbb{C})$ verify (3.3) where $\mu, \nu$ satisfy (3.4).

There exists a quasi-conformal map $\chi$ from $B_{1}(0)$ into itself and a holomorphic function $F$ on $\chi(D)$ such that

$$
f=F \circ \chi .
$$

Moreover the function $\chi$ and its inverse $\chi^{-1}$ satisfy the following conditions:

$$
\begin{array}{ll}
|\chi(x)-\chi(y)| \leq C|x-y|^{\alpha} & \forall x, y \in B_{1} \\
\left|\chi^{-1}(x)-\chi^{-1}(y)\right| \leq C|x-y|^{\alpha} & \forall x, y \in B_{1}
\end{array}
$$


where $C$ and $\alpha, 0<\alpha<1$, depend upon $k$ only.

Proof. See [B-N, page 116].

Let us define, as in $[\mathrm{A}-\mathrm{M}]$, geometric critical points of solutions of elliptic equations like (3.1). That is, given $u$ as in Proposition 3.1, let $v$ be its stream function and let $\chi$ and $F$, respectively, be the quasi-conformal map and the holomorphic function appearing in the representation (3.8) for $f=u+i v$.

A point $z \in \Omega$ is called a geometric critical point of $u$ if $\chi(z)$ is a critical point (in the standard sense) for $\Re F$. This definition does not depend on the choice of the representation.

According to $[\mathrm{A}-\mathrm{M}]$, we define the geometric index of $u$ at $z \in \Omega$ as the winding number of $F^{\prime}$ at $\chi\left(z_{0}\right)$.

Remark 3.3. We wish to stress that the representation theorem, 3.2, gives us that, up to the change of coordinates $\chi, v$ can be viewed as the harmonic conjugate to $u$. In particular we have that, with respect to the metric in $\chi(D)$, the level lines of $v$ are lines of steepest descent of $u$ and vice versa. Consequently we have that, away from the discrete set of geometric critical points, $u$ is strictly monotone on each connected component of the level lines of $v$, and vice versa.

The following theorem shows that, although the domain $\Omega \backslash \sigma$ is doubly connected, for the particular case of solutions to (1.1) a single valued global stream function $v$ exists.

TheOrem 3.4. Let $u$ be a weak solution to (1.1) with $\psi \in L^{2}(\partial \Omega), \int_{\partial \Omega} \psi=0$.

There exists, and it is unique up to an additive constant, a global stream function $v \in W^{1,2}(\Omega \backslash \sigma)$ related to $u$.

Moreover $v$ is a weak solution of the following Dirichlet-type boundary value problem:

$$
\begin{cases}\operatorname{div}(B \nabla v)=0 & \text { in } \Omega \backslash \sigma, \\ v=\text { const } & \text { on } \sigma, \\ v=\Psi & \text { on } \partial \Omega, \\ \int_{\partial \Omega} B \nabla v \cdot \nu=0, & \end{cases}
$$

where $\Psi=\int \psi \mathrm{d} s$ on $\partial \Omega$.

Here, as above, $B=\left(\operatorname{det}(A)^{-1}\right) A^{T}$. Observe that the constant value of $v$ on $\sigma$ is part of the unknowns of the problem (3.10) and that its weak formulation is to find $v \in W^{1,2}(\Omega)$ such that $v=$ constant on $\sigma, v=\Psi$ on $\partial \Omega$ in the sense of traces and satisfies

$$
\int_{\Omega} B \nabla v \cdot \nabla \varphi=0 \quad \text { for every } \varphi \in W^{1,2}(\Omega) \text { such that } \varphi=\text { constant on } \sigma .
$$

Proof. The reader is referred to [A-DV, Proposition 2.1].

We note that the above theorem applies in particular to $u_{1}, u_{2}$ given by $(2.11)$ and to any linear combination of such solutions. Let $a, b$ be any two real numbers such that $a^{2}+b^{2}=1$ and let us define

$$
\begin{aligned}
& u=a u_{1}+b u_{2}, \quad v=a v_{1}+b v_{2}, \\
& \psi=a \psi_{1}+b \psi_{2}, \quad \Psi=a \Psi_{1}+b \Psi_{2} .
\end{aligned}
$$

Clearly, $u$ is the weak solution to (1.1) and $v$ is its stream function, solving (3.10). When $\sigma$ is replaced with $\sigma^{\prime}$, we define $u^{\prime}, v^{\prime}$ in the same fashion. 
Remark 3.5. Observe that, by (2.9) we have

$$
|\Psi(z)-\Psi(w)| \leq 2 \Gamma_{1}|z-w|^{1 / 2} \quad \text { for every } z, w \in \partial \Omega .
$$

Moreover, by $(2.4),(2.5)$ one easily obtains that there exist points $\tilde{P}, \tilde{Q} \in \partial \Omega$ such that $\Psi$ is monotone on the two simple curves forming $\partial \Omega \backslash\{\tilde{P}, \tilde{Q}\}$. Finally note that

$$
\operatorname{osc}_{\partial \Omega} \Psi=|\Psi(\tilde{P})-\Psi(\tilde{Q})| \geq 1 / \sqrt{2} .
$$

In order to distinguish the one-sided limits as $z \rightarrow \sigma, z \in \Omega \backslash \sigma$, it is convenient to figure out $\sigma$ as a degenerate closed curve. More precisely we present Definition 3.6.

DEFINITION 3.6. Let $\tilde{\sigma}$ be the abstract simple closed curve obtained from two copies of $\sigma$ and gluing two by two the corresponding endpoints. We denote by $\tilde{\Omega}$ the compact manifold obtained by the appropriate gluing of $\bar{\Omega} \backslash \sigma$ with $\tilde{\sigma}$ and by $\tilde{d}$ the geodesic distance on $\tilde{\Omega}$.

For any $d, p>0$, we denote

$$
\Omega_{d, p}=\{z \in \Omega \mid \operatorname{dist}(z, \partial \Omega)>d, \operatorname{dist}(z, \sigma)>p\} .
$$

Proposition 3.7. Let $f=u+i v$, where $u$, $v$ are given by (3.11). We have the following conditions:

(i) $v$ satisfies the Hölder estimate

$$
\left|v\left(z_{1}\right)-v\left(z_{2}\right)\right| \leq C_{1}\left|z_{1}-z_{2}\right|^{\alpha_{1}} \quad \text { for every } z_{1}, z_{2} \in \bar{\Omega}
$$

(ii) $u$ satisfies the estimate

$$
\left|u\left(z_{1}\right)-u\left(z_{2}\right)\right| \leq C_{2}\left(\tilde{d}\left(z_{1}, z_{2}\right)\right)^{\alpha_{1}} \quad \text { for every } z_{1}, z_{2} \in \tilde{\Omega} .
$$

(iii) $f$ is a quasi-conformal mapping on $\Omega \backslash \sigma$.

(iv) $f$ satisfies the lower bound

$$
\left|f\left(z_{1}\right)-f\left(z_{2}\right)\right| \geq C_{3}(d) p^{4 / \alpha_{1}}\left|z_{1}-z_{2}\right|^{1 / \alpha_{1}} \quad \text { for every } z_{1}, z_{2} \in \Omega_{d, p} .
$$

Here $C_{1}, C_{2}, \alpha_{1}>0$ depend on the a priori data only, whereas $C_{3}(d)>0$ depends on the a priori data and on $d$ only.

Remark 3.8. It is useful to stress the difference between the estimates (3.15), (3.16). In fact, since $v$ attains to a constant Dirichlet data on $\sigma$, it is expected that $v$ is continuous across $\sigma$. This is not the case for $u$, which may have different one-sided limits on $\sigma$. This is the main motivation for the introduction of the metric $\tilde{d}$.

The proof of Proposition 3.7 will be given through several steps. At several stages we shall use the change of coordinates described below.

LEMMA 3.9. Let $\Omega$ be a simply connected bounded open set which verifies (2.2) and let $\sigma$ be a curve in $\Omega$ which satisfies (2.3). Then there exists a sense-preserving bi-Lipschitz map $\chi$ from $\Omega \backslash \sigma$ onto $B_{2} \backslash \overline{B_{1}}$, such that the $W^{1, \infty}$ norm of $\chi$ and its inverse are dominated by constants depending on the a priori data only.

Here and in the following we say that $\chi$ is bi-Lipschitz if it is a homeomorphism such that $\chi$ and its inverse belong to $W^{1, \infty}$.

Proof (sketch). First, by locally deforming $\partial \Omega$ and $\sigma$ one can construct a biLipschitz mapping $\chi_{1}$ from $\Omega$ onto a simply connected domain $\Omega_{1}$ with $C^{\infty}$ boundary 
such that $\sigma_{1}=\chi_{1}(\sigma)$ is a $C^{\infty}$ simple curve. Second, one can find a $C^{\infty}$ diffeomorphism $\chi_{2}$ from $\Omega_{1}$ onto the disk $B_{2}(0)$ such that $\sigma_{2}=\chi_{2}\left(\sigma_{1}\right)$ is the segment $\{y=$ $0,|x| \leq 1 / 2\}$. Next, one constructs a bi-Lipschitz mapping $\chi_{3}$ from the upper half disk $\overline{B_{2}^{+}(0)}=\{|z| \leq 2, y \geq 0\}$ onto the half annulus $\overline{B_{2}^{+}(0)} \backslash B_{1}^{+}(0)=\{1 \leq|z| \leq 2, y \geq 0\}$ in such a way that $\chi_{3}\left(\sigma_{2}\right)$ is the inner half circle $\{|z|=1, y \geq 0\}$ and $\chi_{3}$ is the identity mapping on the rest of the boundary. Finally one can extend $\chi_{3}$ as a mapping from $B_{2} \backslash \sigma_{2}$ onto $B_{2} \backslash \overline{B_{1}}$ by symmetry. One can make sure that for each $\chi_{i}, i=1,2,3$, the Jacobian and its inverse are uniformly bounded by constants depending on the a priori data only. In conclusion we pick $\chi=\chi_{3} \circ \chi_{2} \circ \chi_{1}$.

Proof of Proposition 3.7(i). Let $\chi$ be the bi-Lipschitz map constructed in Lemma 3.9 and let us call

$$
\tilde{f}(z)=f \circ \chi^{-1}, \quad z \in B_{2} \backslash \overline{B_{1}} .
$$

By the $W^{1, \infty}$ bounds on $\chi$ and its inverse obtained in Lemma 3.9, $\chi$ is also quasi-conformal; hence we can find $\tilde{\mu} \in L^{\infty}\left(B_{2} \backslash \overline{B_{1}}\right)$ such that

$$
\tilde{f}_{\bar{z}}=\tilde{\mu} \tilde{f}_{z} \quad \text { almost everywhere in } B_{2} \backslash \overline{B_{1}},
$$

where

$$
\tilde{\mu} \leq \tilde{k}<1
$$

and $\tilde{k}$ depends on the a priori data only.

Let $\tilde{v}=v \circ \chi^{-1}=\Im \tilde{f}$; then $\tilde{v}$ is a weak solution to

$$
\begin{cases}\operatorname{div}(\tilde{B} \nabla \tilde{v})=0 & \text { in } B_{2} \backslash \overline{B_{1}}, \\ \tilde{v}=\text { const } & \text { on } \partial B_{1}, \\ \tilde{v}=\Psi \circ \chi^{-1} & \text { on } \partial B_{2}, \\ \int_{\partial B_{2}} \tilde{B} \nabla \tilde{v} \cdot \nu=0, & \end{cases}
$$

where $\tilde{B}$ satisfies uniform ellipticity bounds of the type (2.10), with constants depending on the a priori data only.

Since the Dirichlet data in (3.21) are given as Hölder continuous traces of a $W^{1,2}\left(B_{2} \backslash \overline{B_{1}}\right)$ function, by standard results of regularity up to the boundary, we obtain that $\tilde{v}$ satisfies a uniform Hölder estimate in $\overline{B_{2}} \backslash B_{1}$, with constants depending on the a priori data only.

Hence by recalling $v=\tilde{v} \circ \chi,\left.\tilde{v}\right|_{\partial B_{1}}=\left.v\right|_{\sigma}=$ constant, and by the estimate

$$
\left|\chi\left(z_{1}\right)-\chi\left(z_{2}\right)\right| \leq C_{4} \tilde{d}\left(z_{1}, z_{2}\right) \quad \text { for every } z_{1}, z_{2} \in \Omega \backslash \sigma,
$$

following from Lemma 3.9, (3.15) follows.

Proof of Proposition 3.7(ii). Let us apply the representation Theorem 3.2 to $\tilde{f}$, which gives us that, up to a quasi-conformal change of coordinates, $\tilde{u}=u \circ \chi^{-1}$ is the conjugate function to $-\tilde{v}$.

Hence by a local use of Privaloff's Theorem (see, e.g., [B-J-S, Part II, Chapter 6, Theorem 5, page 279]) we obtain that also $\tilde{u}$ satisfies a uniform Hölder estimate in $\overline{B_{2}} \backslash B_{1}$, with constants only depending on the a priori data. Hence (3.16) follows from (3.22).

In order to proceed with the proof of (iii) of Proposition 3.7 we shall need the following two lemmas. 
Let us extend $\tilde{f}, \tilde{\mu}$ to $B_{2} \backslash \overline{B_{1 / 2}}$ by the reflection rules

$$
\left\{\begin{array}{l}
\tilde{f}(z)=\overline{\tilde{f}(1 / \bar{z})}+2 c i, \quad z \in B_{2} \backslash \overline{B_{1 / 2}}, \\
\tilde{\mu}(z)=\overline{\tilde{\mu}(1 / \bar{z}),}
\end{array}\right.
$$

where $c=\left.\tilde{v}\right|_{\partial B_{1}}$.

We obtain that $\tilde{f} \in W^{1,2}\left(B_{2} \backslash \overline{B_{1 / 2}}, \mathbb{C}\right)$ and satisfies (3.19) on all of $B_{2} \backslash \overline{B_{1 / 2}}$ where $|\tilde{\mu}| \leq \tilde{k}<1$ obviously holds throughout. Note that (3.23) imply that $\tilde{u}, \tilde{v}$ satisfy the reflection rules

$$
\left\{\begin{array}{l}
\tilde{u}(z)=\tilde{u}(1 / \bar{z}), \\
\tilde{v}(z)=2 c-\tilde{v}(1 / \bar{z}), \quad z \in B_{2} \backslash \overline{B_{1 / 2}},
\end{array}\right.
$$

and according to Proposition 3.1 are solutions to uniformly elliptic equations in all of $B_{2} \backslash \overline{B_{1 / 2}}$.

LEMMA 3.10. u has exactly two geometric critical points $\tilde{P}_{1}, \tilde{P}_{2}$ of index one in $B_{2} \backslash \overline{B_{1 / 2}}$. $\tilde{P}_{1}, \tilde{P}_{2}$ belong to $\partial B_{1}$ and they are distinct.

Remark. It may be useful to stress that $\tilde{P}_{1}, \tilde{P}_{2}$ are also the unique geometric critical points of $\tilde{v}$ in $B_{2} \backslash \overline{B_{1 / 2}}$.

Proof. This statement is proven in [A-DV, Proposition 3.2] except from the fact that $\tilde{P}_{1}, \tilde{P}_{2}$ are distinct. This can be obtained by the following contradiction argument, if we had $\tilde{P}_{1}=\tilde{P}_{2}$ then, on $\partial B_{1} \backslash\left\{\tilde{P}_{1}\right\}, \tilde{v} \equiv$ constant and hence $\tilde{u}$ should be strictly monotone along such a simple curve, thus contradicting its continuity at $\tilde{P}_{1}$.

Let us denote $m=\min _{\partial \Omega} \Psi, M=\max _{\partial \Omega} \Psi$, and $c=\left.v\right|_{\sigma}$. Observe that by the use of the maximum principle in $\left(3.10^{\prime}\right)$ one obtains $m<c<M$.

Lemma 3.11. For any $t \in(m, M), t \neq c$, the level line $\{z \in \Omega \backslash \sigma \mid v(z)=t\}$ is composed by a simple curve $\gamma_{t}$ joining the two connected components of the level set $\{z \in \partial \Omega \mid \Psi(z)=t\}$.

The level line $\{z \in \Omega \backslash \sigma \mid v(z)=c\}$ is composed of two simple curves $\gamma_{c}^{1}$, $\gamma_{c}^{2}$ each joining $\sigma$ with one of the two connected components of $\{z \in \partial \Omega \mid \Psi(z)=c\}$, respectively. Moreover the limit points of $\gamma_{c}^{1}, \gamma_{c}^{2}$ on $\sigma$ are given by two single points $P_{1}, P_{2}$ which are distinct as elements of $\tilde{\sigma}$.

Proof. By the continuity (3.15) of $v$ we have that for every $t \in(m, M)$ the limit points of $\{z \in \Omega \backslash \sigma \mid v(z)=t\}$ on $\partial \Omega \cup \tilde{\sigma}$ all belong to $\{z \in \partial \Omega \mid \Psi(z)=t\}$ if $t \neq c$ and to $\{z \in \partial \Omega \mid \Psi(z)=c\} \cup \tilde{\sigma}$ if $t=c$.

Let $t \neq c$ and let $z_{0} \in \Omega \backslash \sigma$ be such that $v\left(z_{0}\right)=t$. By Lemma 3.10 we have that $v=\tilde{v} \circ \chi$ has no geometric critical points in $\Omega \backslash \sigma$. Therefore, by the maximum principle, the connected component $\gamma_{t}$ of $\{v=t\}$ containing $z_{0}$ is a simple curve having endpoints on $\partial \Omega$. Again, by the maximum principle, we obtain that $v \neq t$ outside of $\gamma_{t}$ and hence $\{v=t\}=\gamma_{t}$. By the same reasoning, we may find two distinct $\operatorname{arcs} \gamma_{c}^{1}, \gamma_{c}^{2}$ in $\Omega \backslash \sigma$ on which $v=c$, each joining $\sigma$ to the two distinct components of $\{z \in \partial \Omega \mid \Psi(z)=c\}$. Such curves disconnect $\Omega \backslash \sigma$, and hence, by the maximum principle, they exhaust the level set $\{v=c\}$. Concerning the limit points of $\{v=c\}$ on $\sigma$, these coincide with the preimages through $\chi$ of the geometric critical points $\tilde{P}_{1}$, $\tilde{P}_{2}$ of $\tilde{v}$, and the thesis follows.

Proof of Proposition 3.7(iii). It suffices to prove that $f$ is univalent. We use the notation introduced in Lemma 3.11. Let $\tilde{\sigma}_{1}, \tilde{\sigma}_{2}$ the abstract simple curves forming $\tilde{\sigma} \backslash\left\{P_{1}, P_{2}\right\}$. Using the representation $u=\tilde{u} \circ \chi$ and the absence of geometric critical points for $\tilde{u}$ in $B_{2} \backslash\left(\overline{B_{1 / 2}} \cup\left\{\tilde{P}_{1}, \tilde{P}_{2}\right\}\right)$ we have that $u$ is strictly increasing on each of 
the curves $\gamma_{c}^{1} \cup \gamma_{c}^{2} \cup \tilde{\sigma}_{i}, i=1,2$. Analogously, when $t \in(m, M), t \neq c, u$ is strictly increasing on $\gamma_{t}$. Therefore for any $\zeta=s+i t \in f(\Omega \backslash \sigma)$ there exists a unique $z \in \Omega \backslash \sigma$ such that $v(z)=t, u(z)=s$.

Proof of Proposition 3.7(iv). With the aid of Theorem 3.2 and of a suitable conformal mapping, we obtain that there exist $R>1$ depending on the a priori data only and a quasiconformal mapping $\chi_{1}$ from $B_{2} \backslash \overline{B_{1 / 2}}$ onto $B_{R} \backslash \overline{B_{1}}$ and a holomorphic function $F$ on $B_{R} \backslash \overline{B_{1}}$ such that

$$
\tilde{f}=F \circ \chi_{1}
$$

moreover, $\chi_{1}, \chi_{1}^{-1}$ satisfy uniform Hölder estimates with constants depending on the a priori data only.

Let $U, V$ be the real and imaginary part of $F$, respectively.

We remark that, in view of Lemma 3.10, $F$ has exactly two critical points, which are distinct and have multiplicity one, in $B_{R} \backslash \overline{B_{1}}$. We denote such points $\zeta_{1}=\chi_{1}\left(\tilde{P}_{1}\right)$, $\zeta_{2}=\chi_{1}\left(\tilde{P}_{2}\right)$. Let us denote $D=B_{R} \backslash \overline{B_{1}}$ and $D_{d}=B_{R-d} \backslash \overline{B_{1+d}}, d>0$. We claim the following lower bound on $\left|F^{\prime}\right|$, whose proof is deferred to the end of this section.

Claim. There exists a positive constant $C_{5}$ depending on the a priori data and on $d$ only such that the following estimate holds

$$
\left|F^{\prime}(z)\right| \geq C_{5}\left|z-\zeta_{1}\right|\left|z-\zeta_{2}\right| \quad \text { for any } z \in D_{d}
$$

Let us now recall that $F=f \circ \chi^{-1} \circ \chi_{1}^{-1}$ and let us fix $d, p>0$. Denote by $\gamma$ the image through $\chi_{1}$ of $\partial B_{1}$, that is, $\gamma=\left(\chi_{1} \circ \chi\right)(\tilde{\sigma})$. Let $\alpha_{2}>0$ be a uniform Hölder exponent for $\chi_{1} \circ \chi$ and its inverse. We recall that $\alpha_{2}$ depends on the a priori data only.

For any $z \in \Omega_{d, p}$ we have

$$
\begin{aligned}
& \operatorname{dist}\left(\chi_{1} \circ \chi(z), \partial B_{R}\right) \geq C_{6} d^{1 / \alpha_{2}}, \\
& \operatorname{dist}\left(\chi_{1} \circ \chi(z), \gamma\right) \geq C_{6} p^{1 / \alpha_{2}},
\end{aligned}
$$

where $C_{6}$ depends on the a priori data only.

We remark that $F(\gamma)=f(\sigma)$ is a horizontal segment $l$.

So using (3.25) we can show that the image through $f$ of $\Omega_{d, p}$ is contained in a doubly connected open set $D_{1} \subset f(\Omega \backslash \sigma)$ whose boundary is constituted by two curves $\gamma_{1}$ and $\gamma_{2}$. The outer one, $\gamma_{1}$, is a Jordan curve such that for any $z_{0}, z_{1} \in \gamma_{1}$ the following estimate holds

$$
\operatorname{length}_{\gamma_{1}}\left(z_{0}, z_{1}\right) \leq C_{7}\left|z_{0}-z_{1}\right| .
$$

On the other hand, $\gamma_{2}$ is the set of points whose distance from the segment $l$ is equal to $C_{8} p^{3 / \alpha_{2}}$.

Furthermore on $D_{1}$ we can find the following estimate:

$$
\left|\left(F^{-1}\right)^{\prime}\right|(z) \leq C_{9} p^{-3 / \alpha_{2}} \quad \text { for any } z \in D_{1} .
$$

Then, evaluating the geodetic distance on $D_{1}$, we have that for any $z, w \in D_{1}$ it holds that

$$
\left|F^{-1}(z)-F^{-1}(w)\right| \leq C_{10} p^{-4 / \alpha_{2}}|z-w| ;
$$

hence for any $z, w \in \Omega_{d, p}$ we have

$$
|f(z)-f(w)| \geq C_{11} p^{4 / \alpha_{2}}|z-w|^{1 / \alpha_{2}} .
$$


The constants $C_{7}-C_{11}$ depend on $d$ and on the a priori data only. So (3.17) follows.

Proof of the Claim. We adapt arguments used in [A1, Theorem 1.3]. First, we notice that $F$ is Hölder continuous in $D$. Hence $|F|$ can be bounded on $D$ by a constant $C_{12}, C_{12}$ depending on the a priori data only, and in view of (3.14) there exists $d_{1}$ small enough such that for any $0<d \leq d_{1}$ the oscillation of $V$ on $\partial D_{d}$ is greater than $1 / 2 \sqrt{2}$.

Without loss of generality we can restrict our attention to the case $0<d \leq d_{1}$; then, by using estimates on Cauchy's integrals, we have

$$
\begin{array}{cc}
\left|F^{\prime}\right| \leq 2 C_{12} / d \quad \forall z \in D_{d / 2}, \\
\left|F^{\prime \prime}\right| \leq 8 C_{12} / d^{2} \quad \forall z \in D_{d / 2} .
\end{array}
$$

We denote $\phi=\log \frac{\left|F^{\prime}\right|}{\left|z-\zeta_{1}\right|\left|z-\zeta_{2}\right|}$; this is a harmonic function in $D$. Let $M=$ $\sup _{D_{d / 2}} \phi$; then we apply the Harnack inequality to $M-\phi$ and obtain

$$
\sup _{D_{d}}(M-\phi) \leq c \inf _{D_{d}}(M-\phi),
$$

where $c$ depends on $d$ and on $R$ only. This, in turn, implies that

$$
\inf _{D_{d}} \phi \geq M-c\left(M-\sup _{D_{d}} \phi\right)
$$

Notice that we have

$$
1 / 2 \sqrt{2} \leq \operatorname{osc}_{\partial D_{d}} V \leq C_{13} \max _{D_{d}}\left|F^{\prime}\right| \leq C_{14} \max _{D_{d}} \exp \phi,
$$

and hence $M \geq C_{15}>0$. Using (3.29), possibly choosing a smaller value for the constant $d_{1}$, we can find an upper bound on $M-\sup _{D_{d}} \phi$. Hence we can find a constant $C_{16}$, depending on the a priori data and on $d$ only, such that $\inf _{D_{d}} \phi \geq C_{16}$ and the claim follows.

4. Stability for a Cauchy problem. Let $u$ be given by (3.11) and let $u^{\prime}$ be given accordingly when $\sigma$ is replaced with $\sigma^{\prime}$. Let $v$ and $v^{\prime}$ be the stream functions of $u$ and $u^{\prime}$, respectively; we choose to normalize $v, v^{\prime}$ in such a way that they have the same Dirichlet data $\Psi$ on $\partial \Omega$.

Let us denote $\Phi=W+i Z=u-u^{\prime}+i\left(v-v^{\prime}\right): \Omega \backslash\left(\sigma \cup \sigma^{\prime}\right) \mapsto \mathbb{C}$.

We have that $Z$ is identically zero on $\partial \Omega$ and $|W| \leq \sqrt{2} \varepsilon$ on $\Sigma$. We remember that, by Proposition 3.7(i), (ii), there exists a constant $K_{1}$ depending on the a priori data only such that

$$
|\Phi(z)| \leq K_{1} \quad \text { for any } z \in \Omega \backslash\left(\sigma \cup \sigma^{\prime}\right) .
$$

Furthermore by (3.15) the function $Z$ is Hölder continuous on $\bar{\Omega}$ with constants depending on the a priori data only.

$\Phi$ satisfies the Cauchy problem

$$
\begin{cases}\Phi_{\bar{z}}=\mu \Phi_{z}+\nu \overline{\Phi_{z}} & \text { in } \Omega \backslash\left(\sigma \cup \sigma^{\prime}\right), \\ |\Phi| \leq \sqrt{2} \varepsilon & \text { on } \Sigma, \\ \Im \Phi=0 & \text { on } \partial \Omega,\end{cases}
$$

where $|\mu|+|\nu| \leq k<1$. 
We want to estimate $|Z|$ on $\bar{\Omega}$ in terms of $\varepsilon$.

Proposition 4.1. Under the previous assumptions we have

$$
|Z(z)| \leq \eta(\varepsilon) \quad \text { for any } z \in \bar{\Omega},
$$

where $\eta$ is a positive function defined on $(0,+\infty)$ that verifies

$$
\eta(\varepsilon) \leq K_{2}(\log |\log \varepsilon|)^{-\beta_{1}} \quad \text { for every } \varepsilon, 0<\varepsilon<1 / e .
$$

Here $K_{2}$ and $\beta_{1}$ are positive constants depending on the a priori data only.

Let us recall some notions from potential theory; see for instance the book by J. Heinonen, T. Kilpeläinen, and O. Martio, [H-K-Ma].

Let $D$ be a bounded open set. Let $A \in L^{\infty}(D)$ be a $2 \times 2$ matrix which satisfies (2.10).

We denote by $\mathcal{L}_{A}$ the differential operator

$$
\mathcal{L}_{A} u=-\operatorname{div}(A \nabla u) .
$$

Definition 4.2. A function $u: D \mapsto \mathbb{R} \cup\{+\infty\}$ is called $\mathcal{L}_{A}$-superharmonic in $D$ if

(i) $u$ is lower semicontinuous;

(ii) $u \not \equiv+\infty$ in any connected component of $D$;

(iii) for any open set $D_{1} \subset \subset D$ and any $h \in C\left(\overline{D_{1}}\right)$, such that $\mathcal{L}_{A} h=0$ in the weak sense in $D_{1}$, if $u \geq h$ on $\partial D_{1}$ then $u \geq h$ in $D_{1}$.

A function $u$ is $\mathcal{L}_{A}$-subharmonic in $D$ if $-u$ is $\mathcal{L}_{A}$-superharmonic in $D$.

DeFINITION 4.3. Let $E$ be a subset of $\partial D$ and let $\chi_{E}$ be its characteristic function. We define the $\mathcal{L}_{A}$-harmonic measure of $E$ with respect to $D$ as the upper Perron solution with respect to $\chi_{E}$; that is,

$$
\omega(z)=\omega\left(E, D, \mathcal{L}_{A} ; z\right)=\inf \left\{u(z) \mid u \in \mathcal{U}_{E}\right\} \quad \text { for any } z \in D,
$$

where $\mathcal{U}_{E}$ is the class of the $\mathcal{L}_{A}$-superharmonic functions $u$ in $D$ such that $u \geq 0$ and $\liminf _{x \rightarrow y} u(x) \geq \chi_{E}(y)$ for any $y \in \partial D$.

Lemma 4.4. Let $D$ be a bounded domain. Let $f \in W^{1,2}(D, \mathbb{C})$ satisfy (3.3), (3.4). There exists a $2 \times 2$ matrix $A_{1} \in L^{\infty}(D)$ satisfying (2.10) with constants $\lambda, \Lambda$ depending on $k$ only such that $\phi=\log |f|$ is $\mathcal{L}_{A_{1}}$-subharmonic.

Proof. Let $z$ be a point in $D$ such that $f(z) \neq 0$. Locally, on a neighborhood of $z$, we can define the function $\phi_{1}=\log f$ where $\log$ is any possible determination of the logarithm in the complex plane.

In this neighborhood $\phi_{1}$ verifies the equation

$$
\left(\phi_{1}\right)_{\bar{z}}=\mu\left(\phi_{1}\right)_{z}+\nu_{1} \overline{\left(\phi_{1}\right)_{z}}
$$

where $\nu_{1}=\nu \bar{f} / f$ and hence $|\mu|+\left|\nu_{1}\right| \leq k<1$.

Then we consider the matrix $A_{1}$ corresponding to $\mu$ and $\nu_{1}$, as in (3.7). By Proposition 3.1 the function $\phi=\log |f|=\Re \log f$ locally verifies

$$
\operatorname{div}\left(A_{1} \nabla \phi\right)=0
$$

in the weak sense.

We remark that we can define $\phi=\log |f|$ globally as a $W_{l o c}^{1,2}\left(D_{1}\right)$ function, where $D_{1}=\{z \in D \mid f(z) \neq 0\}$; hence using a partition of unity it is easy to show that (4.7) holds weakly in $D_{1}$. 
Clearly the set $\{z \in D \mid f(z)=0\}$ consists of isolated points and $\phi$ goes uniformly to $-\infty$ as $z$ converges to an element of such a set.

Using this remark and the maximum principle, we can prove in an elementary way that $\phi=\log |f|$ is $\mathcal{L}_{A_{1}}$-subharmonic.

By the use of suitable $\mathcal{L}_{A_{1}}$-harmonic measure we obtain a Hölder stability estimate in the interior for Cauchy problems like (4.2), as follows.

THEOREM 4.5. Let $D$ be bounded domain and $E$ a subset of $\partial D$. Let $f$ satisfy (3.3), (3.4).

If $C=\sup |f|$ on $D$ and we have that, given $\varepsilon>0$,

$$
\limsup _{x \rightarrow y}|f(x)| \leq \varepsilon
$$

for any $y \in E$, then for any $z \in D$ the following estimate holds

$$
|f(z)| \leq C^{1-\omega(z)} \varepsilon^{\omega(z)},
$$

where $\omega=\omega\left(E, D, \mathcal{L}_{A_{1}}\right)$ is the $\mathcal{L}_{A_{1}}$-harmonic measure of $E$ with respect to $D$ and the matrix $A_{1}$ is defined as in the thesis of the Lemma 4.4.

Proof. We can assume, without loss of generality, that $0<\varepsilon<C$. Consider the function $\phi=\log |f|$, by the fact that Lemma $4.4 \phi$ is $\mathcal{L}_{A_{1}}$-subharmonic. Let $\omega=\omega\left(E, D, \mathcal{L}_{A_{1}}\right)$ be the $\mathcal{L}_{A_{1}}$-harmonic measure of $E$ with respect to $D$.

Let us denote $\phi_{2}=\frac{\phi-\log (C)}{\log (\varepsilon)-\log (C)}$. It is easy to see that $\phi_{2}$ belongs to the upper class $\mathcal{U}_{E}$. Hence for any $z \in D$ we have $\omega(z) \leq \phi_{2}(z)$ and so

$$
\phi(z) \leq \log (\varepsilon)(\omega(z))+\log (C)(1-\omega(z)) .
$$

And this clearly implies the thesis.

Remark. Observe that in view of Proposition 3.1 the above Theorem 4.5 could be restated in terms of a Cauchy problem for an elliptic equation like (3.1).

Proof of Proposition 4.1 (Sketch). The proof of this proposition can be obtained along the same lines as in the proof of Theorem 3.1 in [A2], once Theorem 4.5 is available.

First consider curves $\gamma$, with the first endpoint on $\Sigma$, whose $h$-neighborhoods $\gamma_{h}$ are contained in $\Omega \backslash\left(\sigma \cup \sigma^{\prime}\right)$.

Then we apply Theorem 4.5 inside such domains $\gamma_{h}$, and we consider a point $z \in \gamma_{h}$ and $\omega=\omega\left(\Sigma \cap \partial \gamma_{h}, \gamma_{h} ; \mathcal{L}_{A_{1}}\right)$ as in Theorem 4.5. We obtain, recalling (4.1), $(4.2)$,

$$
|\Phi(z)| \leq K_{1}^{1-\omega(z)} \varepsilon^{\omega(z)} .
$$

We find a positive lower bound on $\omega(z)$ by a repeated use of the Harnack inequality; then through Hölder continuity of $Z$ in $\bar{\Omega}$ we can evaluate an upper bound for $|Z|$ on $\overline{\gamma_{h}}$. Finally we use the maximum principle together with the fact that $v$ and $v^{\prime}$ are constant on $\sigma, \sigma^{\prime}$, respectively, to obtain the desired bound for $|Z|$ on $\bar{\Omega}$.

5. Proof of the main Theorem 2.1. The proof of Theorem 2.1 will be completed by combining Proposition 4.1 with the following result.

Proposition 5.1. Let all the assumptions of Theorem 2.1 be satisfied with the exception of (2.12). Let $v_{i}$ be the stream functions related to $u_{i}$ and let $v_{i}^{\prime}$ be those related to $u_{i}^{\prime}$. If we have

$$
\max _{i=1,2}\left\|v_{i}-v_{i}^{\prime}\right\|_{L^{\infty}(\Omega)} \leq \eta
$$


then the two cracks $\sigma, \sigma^{\prime}$ satisfy

$$
\mathrm{d}_{H}\left(\sigma, \sigma^{\prime}\right) \leq K_{3} \eta^{\beta_{2}},
$$

where $K_{3}, \beta_{2}, K_{3}>0,0<\beta_{2}<1$, only depend on the a priori data.

Proof. Up to reversing the role of $\sigma$ and $\sigma^{\prime}$ we may fix $z_{0} \in \sigma^{\prime} \backslash \sigma$ in such a way that $p=\operatorname{dist}\left(z_{0}, \sigma\right)=d_{H}\left(\sigma, \sigma^{\prime}\right)>0$.

There exists a positive constant $K_{4}>1$ only depending on the a priori data such that

$(5.3)$ (a) $\quad B_{p / K_{4}}\left(z_{0}\right) \subset \Omega_{\delta / 2} \backslash \sigma$;

(5.3)(b) there exists a point $z_{1} \in \sigma^{\prime}$ such that $\left|z_{1}-z_{0}\right|=p / 2 K_{4}$.

Hence we can determine two real numbers $a, b$ such that $a^{2}+b^{2}=1$ and

$$
a u_{1}\left(z_{o}\right)+b u_{2}\left(z_{0}\right)=a u_{1}\left(z_{1}\right)+b u_{2}\left(z_{1}\right)
$$

holds true.

So we define $u$ and $v$ as in (3.11) and it turns out that

$$
u\left(z_{o}\right)=u\left(z_{1}\right) .
$$

Recall that $u$ solves (1.1) and $v$ is its stream function. Let, as usual, $f=u+i v$.

Then by (iv) of Proposition 3.7 there exists a constant $K_{5}$, depending on the a priori data only, such that

$$
p^{5 / \alpha_{1}} \leq K_{5}\left|f\left(z_{0}\right)-f\left(z_{1}\right)\right| .
$$

Note that, by (5.5), $\left|f\left(z_{0}\right)-f\left(z_{1}\right)\right|=\left|v\left(z_{0}\right)-v\left(z_{1}\right)\right|$. We have that $z_{0}$ and $z_{1}$ belong to $\sigma^{\prime}$; hence $v^{\prime}\left(z_{0}\right)=v^{\prime}\left(z_{1}\right)$.

So we have

$$
\left|f\left(z_{0}\right)-f\left(z_{1}\right)\right| \leq 2 \eta \text {. }
$$

Consequently

$$
p \leq K_{6} \eta^{\alpha_{1} / 5},
$$

where $K_{6}$ and $\alpha_{1}$ only depend on the a priori data.

Concluding remarks. Let us recall that, for the case of uniform background conductivity, a log-log-type stability like the present one was proven in [A2]. Subsequently, in [A3], it was shown that the stability could be improved to a log-type estimate. A $C^{2, \alpha}$ a priori bound on $\sigma$ was assumed. It can be verified that the approach in [A3] could be used with minor adaptations also in the present case, at the cost of assuming a somewhat stronger a priori assumption on the crack. For instance, an analysis of this sort has been developed in [R] where it was assumed a $C^{1, \alpha}$ bound on $\sigma$ and a Lipschitz bound on $A$. Let us stress here that in view of Theorem 4.5 in this paper any regularity assumption on $A$ can be dropped.

Let us recall here also the examples in [A4] for the so-called inverse problem of corrosion detection, which is different, but strictly allied, to the crack problem. Such examples show that logarithmic stability is best possible for that problem and they strongly suggest that this is the case also for the crack problem.

From another point of view, we notice that the Lipschitz regularity assumptions on $\sigma$ and on $\partial \Omega$ could be further relaxed. In fact we could cast our analysis within 
the theory of quasicircles (see $[\mathrm{P}$, Chapter 5] and [L]) and prescribe that $\sigma$ and $\partial \Omega$ satisfy the so-called arc condition. This ensures that quasi-conformal mappings in $\Omega \backslash \tilde{\sigma}$ are Hölder continuous up to the boundary, thus permitting us to derive statements analogous to Proposition 3.7 and, consequently, to Theorem 2.1. However, we have preferred to confine ourselves within the Lipschitz class which, we believe, is sufficiently wide and manageable from the applications point of view.

\section{REFERENCES}

[A1] G. Alessandrini, An identification problem for an elliptic equation in two variables, Ann. Mat. Pura Appl., 145 (1986), pp. 265-296.

[A2] G. AlESSANDRIni, Stable determination of a crack from boundary measurements, Proc. Roy. Soc. Edinburgh Sect. A, 123 (1993), pp. 497-516.

[A3] G. Alessandrini, Stability for the crack determination problem, in Inverse Problems in Mathematical Physics, L. Päivärinta and E. Somersalo, eds., Springer-Verlag, Berlin, Heidelberg, 1993, pp. 1-8.

[A4] G. Alessandrini, Examples of instability in inverse boundary-value problems, Inverse Problems, 13 (1997), pp. 887-897.

[A-DB] G. Alessandrini and E. Di Benedetto, Determining 2-dimensional cracks in 3dimensional bodies: Uniqueness and stability, Indiana Univ. Math. J., 46 (1997), pp. 1-82.

[A-DV] G. Alessandrini and A. Diaz Valenzuela, Unique determination of multiple cracks by two measurements, SIAM J. Control Optim., 34 (1996), pp. 913-921.

[A-M] G. Alessandrini And R. MAGNANini, Elliptic equations in divergence form, geometric critical points of solutions, and Stekloff eigenfunctions, SIAM J. Math. Anal., 25 (1994), pp. 1259-1268.

[B-J-S] L. Bers, F. John, and M. Schechter, Partial Differential Equations, Interscience, New York, London, Sydney, 1964.

[B-N] L. Bers AND L. NiREnBerG, On a representation theorem for linear elliptic systems with discontinuous coefficients and its applications, in Convegno Internazionale sulle Equazioni Lineari alle Derivate Parziali, Trieste, Cremonese, Roma, 1955.

[Br-V] K. BRYAN AND M. Vogelius, A uniqueness result concerning the identification of a collection of cracks from finitely many boundary measurements, SIAM J. Math. Anal., 23 (1992), pp. 950-958.

[DV] A. Diaz Valenzuela, Unicità e stabilità per il problema inverso del crack perfettamente isolante, thesis, Università degli Studi di Trieste, 1993.

[F-V] A. Friedman And M. Vogelius, Determining cracks by boundary measurements, Indiana Univ. Math. J., 38 (1989), pp. 527-556.

[H-K-Ma] J. Heinonen, T. Kilpeläinen, and O. Martio, Nonlinear Potential Theory of Degenerate Elliptic Equations, Clarendon Press, Oxford, New York, Tokyo, 1993.

[K-Se] H. KIm AND J. K. SEO, Unique determination of a finite number of collection of cracks from two boundary measurements, SIAM J. Math. Anal., 27 (1996), pp. 1336-1340.

[L] O. Lehto, Univalent Functions and Teichmüller Spaces, Springer-Verlag, New York, Berlin, Heidelberg, 1987.

[P] Сн. Pommerenke, Boundary Behaviour of Conformal Maps, Springer-Verlag, Berlin, Heidelberg, 1992.

[R] L. Rond, Stabilità per il problema inverso dei crack in un corpo non omogeneo, thesis, Università degli Studi di Trieste, 1996. 